\title{
Las cooperativas de enseñanza: un emprendimiento basado en el liderazgo y formación continua docente
}

\section{The teaching cooperatives: an enterprise based on leadership and continuous teacher training}

\author{
Jesús López Belmonte ${ }^{1}$, Juan Antonio López Núñez ${ }^{2}$, Arturo Fuentes Cabrera ${ }^{3}$ \\ ${ }^{1}$ Universidad Internacional de Valencia, España (jesus.lopezb@campusviu.es) \\ ${ }^{2}$ Universidad de Granada, España (juanlope@ugr.es) \\ ${ }^{3}$ Universidad de Granada, España (arturofuentes@ugr.es)
}

Recibido el 5 de octubre de 2017; revisado el 21 de diciembre de 2017; aceptado el 25 de septiembre de 2018; publicado el 1 de junio de 2019

\section{RESUMEN:}

Las cooperativas de enseñanza son entidades educativas de carácter social que agrupan a un conjunto de miembros, unidos bajo las mismas necesidades, que persiguen la creación y mantenimiento de puestos laborales en tiempos de recesión económica. Pretenden ofrecer un servicio innovador, eficaz y de calidad basado en el liderazgo y formación continua de los profesionales de este tipo de centros escolares. El objetivo del presente estudio se centra en conocer la vertiente empresarial, formativa y de liderazgo del docente cooperativista de la Ciudad Autónoma de Ceuta. Para ello se ha optado por un método cuantitativo de tipo descriptivo, empleando un cuestionario ad hoc para la recogida de datos en una muestra de 68 docentessocios pertenecientes a las 3 cooperativas de enseñanza de dicha ciudad. En base a los resultados, se obtiene una dominancia del género femenino y una edad avanzada entre los docentes-socios de las cooperativas analizadas. Estos profesionales conocen las peculiaridades que presentan estas entidades $y$, aunque confían en la inversión realizada, reflejan carencias burocráticas para la gestión cooperativa. En cuanto al liderazgo, se decantan conocedores de tal fenómeno y le otorgan gran relevancia. Pero, en el plano formativo, presentan deficientes conocimientos empresariales y aunque consideran que la formación continua es importante, la mayoría no suele efectuar más de 2 cursos al año. Se concluye que estos profesionales conforman un perfil femenino de edad avanzada que confía en el liderazgo como fenómeno para dinamizar a la entidad, pero no disponen de los conocimientos necesarios para administrar con éxito este tipo de centros educativos, requiriendo de una formación especializada en el ámbito empresarial.

\section{PALABRAS CLAVE: COOPERATIVISMO EDUCATIVO, LIDERAZGO DOCENTE, FORMACIÓN COMPLEMENTARIA.}

\begin{abstract}
:
Educational cooperatives are educational entities of a social nature that bring together a group of members, united under the same needs, who pursue the creation and maintenance of jobs in times of economic recession. They intend to offer an innovative, effective and quality service based on the leadership and continuous training of professionals of this type of school. The objective of this study is to know the business, training and leadership aspects of the cooperative teacher of the Autonomous City of Ceuta. For this purpose, a quantitative method of descriptive type was chosen, using an ad hoc questionnaire for the collection of data in a sample of 68 teacher-members belonging to the 3 teaching cooperatives of said city. Based on the results, a dominance of the feminine gender and an advanced age among the teachers-partners of the analyzed cooperatives is obtained. These professionals know the peculiarities that these
\end{abstract}


entities present and although they trust in the investment made, they reflect bureaucratic deficiencies for cooperative management. In terms of leadership, experts are aware of this phenomenon and give it great relevance. But, on the training level, they have poor business knowledge and although they consider that continuing education is important, most do not usually do more than 2 courses a year. It is concluded that these professionals make up an elderly female profile that relies on leadership as a phenomenon to energize the entity but do not have the necessary knowledge to successfully manage this type of educational centers, requiring specialized training in the business field.

\section{KEYWORDS: EDUCATIONAL COOPERATIVISM, TEACHING LEADERSHIP, COMPLEMENTARY EDUCATION.}

\section{INTRODUCCIÓN}

Las cooperativas son aquellas entidades sociales que nacen para paliar los efectos negativos surgidos tras periodos económicos adversos y recesivos por los que atraviesa la sociedad en la que vivimos. En España, la fuerte crisis económica ocurrida en los últimos años provocó una alta mortalidad empresarial. Las personas afectadas, como solución y medida ante la elevada tasa de pérdida de puestos de trabajo, optaron por la fórmula del emprendimiento social, donde el fin a alcanzar es el bienestar de los miembros que constituyen estas empresas. Las cooperativas son consideradas las entidades que mejor soportan la conservación del empleo en tiempos económicos difíciles, ya que luchan por el mantenimiento de los puestos de trabajo que se han creado, mediante una gestión eficaz y aprovechamiento de los recursos disponibles (Sanchís, Campos y Mohedano, 2015).

Las consecuencias negativas del desplome económico apenas se percibieron en las cooperativas, puesto que estas están orientadas hacia objetivos sociales, con primacía de las personas involucradas y no sobre el capital, generando una recuperación económica de las regiones donde se implantan. Han demostrado, por tanto, ser viables y prosperar ante situaciones difíciles, con la ayuda y colaboración de todos los miembros que las componen. Estas empresas toman por bandera la innovación, creatividad, flexibilidad, cohesión social, calidad, eficacia, como principales herramientas para afianzar y prolongar la vida empresarial (Cuadrado y Ciruela, 2014). Se centran en la fórmula del autoempleo, por este motivo prosperan en épocas marcadas por una economía negativa y el agotamiento de un mercado que no puede acoger a tanta demanda laboral de personas necesitadas. Son una esperanza ante situaciones inestables y, por ello, se hace necesario la creación de políticas para difundir sus ideales, sobre todo entre los más jóvenes, puesto que su falta de experiencia laboral está directamente relacionada con la marginación a la hora de encontrar un trabajo digno y correspondiente a su grado de formación académica (Gadea y Atxabal, 2015).

Las cooperativas asumen un papel muy importante en la sociedad. Se encargan de satisfacer las necesidades de las personas que las componen y son una magnífica vía para la creación de empleo. Son una fórmula empresarial basada en el trabajo responsable, democrático y asociativo. Se encuentran situadas frente al capitalismo de algunas empresas que toman al capital como fin único a alcanzar; en contraposición a esta postura se encuadran las primeras que entienden al capital conseguido como un simple cauce económico para la consecución de sus fines sociales. Su personal, que trabaja de manera colaborativa, presenta una serie de necesidades comunes que intentan poner solución mediante el esfuerzo y dedicación de cada individuo (Melián y Campos, 2010).

Siguiendo a Frantz y Fonseca (2013), el mundo actual se mueve por intereses económicos pero el cooperativismo, por consiguiente, se centra en promover una economía basada en la satisfacción de las necesidades humanas, aunando los esfuerzos del colectivo bajo una fórmula democrática y participativa, considerando que el esfuerzo de todos dará lugar a la consecución de las metas sociales marcadas por la entidad. Para Cuñat (2015), las cooperativas se representan como aquellas empresas que combinan los objetivos económicos con los sociales y fijan como meta la promoción y mantenimiento de un empleo digno, equitativo y sin discriminación. Y, como tipo de empresa propia de la economía social, contribuyen a la recuperación económica del lugar donde se implantan, puesto que generan empleo de carácter estable y de calidad.

El continuo crecimiento que han experimentado a lo largo de la historia se debe a sus políticas integradoras, democráticas y sociales que las convierten en un medio de subsistencia en tiempos complicados económicamente hablando (Inglada, Sastre y Villarroya, 2015). El modelo cooperativo se encuentra presente en las diferentes zonas de la geografía mundial y concretamente en la mayoría de los sectores, como la sanidad, vivienda, agricultura, 
industria, financiero, consumo y educación, entre otros muchos (Eid y Martínez, 2014).

\subsection{Las cooperativas en el ámbito educativo}

Entrando en materia educativa, la Ley vigente 27/1999 de Cooperativas, en su artículo 103, define a las cooperativas de enseñanza como "aquellas que desarrollen actividades docentes, en sus distintos niveles y modalidades. Y podrán realizar actividades extraescolares así como prestar servicios que faciliten las actividades docentes" (p. 27056).

Las cooperativas de enseñanza son consideradas como una apuesta segura de futuro en el panorama educativo. Son entidades que ofrecen calidad, innovación, eficacia, formación continua y liderazgo en el marco de su proyecto educativo (López, 2017; López y Fuentes, 2018; López, Fuentes y Moreno, 2018).

Estas entidades educativas persiguen formar a las nuevas generaciones discentes en base a los valores sociales y colaborativos que las caracterizan. Se presentan en la sociedad como una posible elección dentro de la oferta de centros educativos, siguiendo un modelo laico e integrador de todas las culturas. Los socios trabajadores que componen este tipo de entidades escolares tienen que convivir con la realización de tareas educativas y empresariales, puesto que son docentes, pero, al mismo tiempo, empresarios de una entidad. Para ello, es muy importante seguir formándose, tanto en aspectos relacionados con la docencia, como en el ámbito de la gestión y dirección de empresas que garantice un futuro prometedor y competente (Inglada, Sastre y Villarroya, 2015; López, Moreno y Pozo, 2018).

Para Egido (2010), la educación en España en los últimos años se caracteriza por ser un medio de difícil acceso laboral, en el que muchos son los candidatos que desean optar por cumplir su vocación docente, pero pocos lo consiguen, habiendo una competencia en formación inicial y complementaria alta. Este panorama está marcado por un gran número de docentes en situación laboral desfavorecida, ya que no han podido acceder a ningún puesto de trabajo relacionado con la docencia o, simplemente, no han tenido acceso a la escuela pública mediante oposición. Este colectivo, sumado a la cifra de jóvenes egresados universitarios de las facultades de educación de España, generan una alta cifra de docentes en nuestro país cada año, contribuyendo a que cada vez resulte más complejo desempeñar la labor docente en un centro educativo.
El gran inconveniente es la escasez de plazas generadas, en contraposición con el gran número de aspirantes que intentan una y otra vez alcanzar un empleo público. Otro punto de similitud es la desmotivación y el estado de frustración al no encontrar ninguna salida laboral tras años de estudios universitarios, planteando incluso la idea de buscar otro tipo de empleo fuera del campo de la educación. Como alternativa y principal salida laboral con proyección de futuro estable de estos profesionales de la educación se establece la fórmula del emprendimiento (Melián y Campos, 2010), es decir, crear su propio centro educativo bajo el amparo de un grupo de personas, unidas por la misma necesidad laboral, que apuestan por el cooperativismo como herramienta para poner solución a esta difícil situación. No será un camino fácil, puesto que tendrán que dedicar mucho esfuerzo y dedicación para empezar una cooperativa de enseñanza desde sus cimientos, tanto jurídicamente como estructuralmente (Fuentes, 2004).

Emprender es un mecanismo que supone desarrollar una idea empresarial más allá del papel. Es un motor de crecimiento de una región, que consiste en darle vida a un producto o servicio necesario para la sociedad. Para ello, es importante desarrollar en el empresario una fuerte iniciativa que dinamice el proyecto. Todo esto supone aceptar un margen de riesgo para, posteriormente, recibir los beneficios correspondientes a la inversión inicial (Ciruela, Cuadrado y Plaza, 2016).

Hoy en día existe una demanda y necesidad creciente acerca de la mejora de la calidad en la educación. Esta es entendida como un concepto intangible que va cambiando con el desarrollo de la sociedad. Por tanto, transferida a la educación, la calidad debe ser entendida como un proceso de desarrollo integral y periódico del docente. Hay que concebir a la educación como un ente vivo que cambia con la sociedad y los docentes no se pueden quedar en el pasado, sino que deben promocionar, ser más competentes y adoptar medidas que contribuyan a forjar la búsqueda e implantación de sistemas de calidad en la educación (Casanova, 2016) y ello solo se consigue a través de la formación continua (Casal y García, 2019). En este sentido, las cooperativas de enseñanza son un fantástico medio para alcanzar la calidad deseada en los procesos de enseñanza y aprendizaje. Los docentes se encuentran ante el reto de formar y preparar para la vida a los futuros ciudadanos y, por tal razón, deben disponer de una formación multidisciplinar (López, Moreno y Pozo, 2018) para 
hacer florecer las competencias y aptitudes en los alumnos para que estos se desenvuelvan con astucia en la sociedad.

En los últimos años, la calidad se ha convertido en un tema relevante para la sociedad. No se concibe un buen producto si este no presenta ciertos indicadores de calidad. En la educación se entiende de igual modo. No podemos ofertar y llevar a cabo procesos de enseñanza eficaces si no están concebidos bajo los preceptos de la calidad (Prieto, 2001).

En el ámbito educativo, la consecución de la calidad debe ser entendida como un trabajo constante y diario en el que participen todos los miembros de la comunidad educativa, como son los profesores, los alumnos y las familias de estos, a través de prácticas democráticas que conlleven a alcanzar dicho vocablo tan deseado en los productos de la sociedad (Muñoz y Murillo, 2010).

Para que las cooperativas de enseñanza, como cualquier otro centro educativo, puedan alcanzar la calidad anhelada, es necesaria la conjugación de dos aspectos fundamentales. Por un lado, debe aparecer el fenómeno del liderazgo dentro de la entidad cooperativa que dinamice y promueva a todo el colectivo hacia la consecución de las metas y, por otro lado, la puesta en marcha de una formación complementaria de los socios que integran estos centros, que contribuya a capacitar a los docentes con mayores competencias y habilidades en materia educativa (López, Fuentes y Moreno, 2018).

$\mathrm{El}$ acelerado ritmo del mundo actual hace que los centros educativos, para satisfacer la demanda de las personas, tengan que implantar sistemas basados en el liderazgo y compromiso del claustro docente (López, 2017), para poder dar respuesta a las necesidades que surgen hoy día entre los usuarios de estos centros con la intención de prestar un servicio de calidad.

Los expertos (Gutiérrez, Gracia y Quiñonez, 2016; Mehdinezhad y Sardarzahi, 2016) consideran que el liderazgo es un fenómeno social destacado hoy en día, que se centra en el conjunto de habilidades y destrezas que dispone una persona para crear vínculos con los miembros que le rodea y ejercer una influencia en ellos. El líder debe adaptarse a cada situación, entorno, lugar en el que se encuentra para poder ejercer tal influencia de forma plena y satisfactoria, aunque no en todas las situaciones se consiguen los mismos resultados, puesto que el ambiente condiciona la actuación del líder. El liderazgo se rige por el principio de voluntariedad, no se puede obligar nadie a seguir a una persona y realizar ciertas acciones, puesto que no obtendríamos el rendimiento deseado.

En esta línea, para prolongar la vida de una entidad, guiar el camino que debe seguir para alcanzar los objetivos y obtener el factor de calidad, resulta imprescindible la figura de una persona que lidere y dirija a la entidad, marcando el rumbo y las pautas a seguir que aseguren la estabilidad en el mercado y las perspectivas de futuro. Esta persona debe ejercer de auténtico líder, influyendo en los miembros de la organización para enriquecer el ambiente, facilitar el alcance de los fines propuestos (Cuevas, Díaz e Hidalgo, 2008).

El líder, por consiguiente, tiene que expandir su papel y sus roles en la organización para conseguir nuevos líderes dentro de un mismo entorno, capaces de movilizar a todo el colectivo sin la necesidad de recaer en una sola persona todo este entramado. Con la aparición de nuevos líderes se asegura la permanencia de este ideario dentro de una entidad, olvidando el temor de que algún día ese líder dejará de serlo o finalizará su etapa laboral (Ferrera, 2013).

En los centros educativos, el liderazgo cobra un aspecto de especial relevancia (Sans, Guàrdia y Triadó, 2016) de naturaleza cooperativa; es preciso disponer de auténticos líderes que animen y motiven a toda la organización, haciendo frente a las distintas adversidades cotidianas que estos centros tienen que solventar cada día en sus ejercicios profesionales (López y Fuentes, 2018).

Pero, no solo basta con liderar a la organización, sino que también hay que formarla y capacitarla. Siguiendo los estudios de Bacaicoa y Marín (2002), la formación complementaria es un aspecto relevante que debe ir encaminado a la consecución de una actualización integral, es decir, tanto en el aspecto didáctico y pedagógico de los conocimientos, como en la rama psicológica que influirá en las relaciones socio-afectivas del entorno escolar, que condicionará el clima desarrollado en el centro educativo.

Según González y Barba (2014), la formación permanente debe ser entendida como un proceso constante de aprendizaje e importante durante toda la vida profesional del profesorado. Estos profesionales deben ser flexibles y aceptar las nuevas metodologías y saberes como una herramienta al alcance del docente para conseguir resultados óptimos en una sociedad bañada por la información y el conocimiento mediado por las TIC (Trujillo, 2015). Para Gisbert y Lázaro (2015), la expansión tecnológica en la sociedad actual ha traído consigo la necesidad de estar al día en aspectos 
innovadores y tecnológicos para no quedar atrapado en las redes de una enseñanza primitiva.

Los alumnos de hoy en día necesitan explorar y adquirir las habilidades necesarias para poder desenvolverse de manera eficaz ante estos nuevos medios que les rodean. En este aspecto, la labor del docente resulta muy necesaria para poder dar respuesta y cubrir los intereses e incertidumbres de los alumnos en materia tecnológica. Para ello, es muy importante que nos encontremos ante un perfil docente, preocupado e interesado por la actualización y renovación de su formación en distintos campos (López, Moreno y Pozo, 2018), así como el desarrollo de sus competencias profesionales (Maciel, 2018).

\section{INVESTIGACIÓN}

Para llevar a cabo el estudio se requiere una arquitectura procedimental que asegure su eficacia sin olvidar ninguno de los elementos que constituyen todo proceso de investigación. El entramado científico que se lleva a cabo se compone de distintos apartados que darán luz a unos resultados que mediante un profundo análisis permitirán establecer las principales conclusiones resultantes de la investigación.

\subsection{Objetivos}

El objetivo principal de esta investigación se centra en conocer la vertiente empresarial, formativa y de liderazgo del docente cooperativista de la Ciudad Autónoma de Ceuta.

Para determinar su grado de alcance se han establecido los siguientes objetivos específicos:

- Conocer el perfil docente de las cooperativas de enseñanza analizadas.

- Averiguar el nivel de conocimiento de los docentes sobre el cooperativismo educacional.

- Determinar el grado de conocimiento del profesorado acerca del fenómeno del liderazgo.

- Conocer el estado de la formación continua realizada por los docentes.

\subsection{Problema}

El principal problema que se presenta en este estudio es comprobar si la figura del docente cooperativista, que normalmente es un profesional titulado en el campo de la educación, está capacitada para liderar una organización caracterizada por el desempeño de funciones de carácter administrativo-pedagógico en la cooperativa. De este problema principal deriva otra cuestión referente a la formación permanente del profesorado; y es si estos realizan cursos complementarios de formación continua en diversos campos de estudio con el fin de lograr una gestión eficaz de la entidad.

\subsection{Metodología}

Para esta investigación se ha optado por la elección de una metodología de corte cuantitativo de tipo descriptivo basado en el estudio estadístico de los datos (McMillan y Schumacher, 2005). Los motivos que han declinado la postura hacia la elección de este método son, por un lado, el hecho de poder estudiar la realidad que viven los docentes-socios de las cooperativas de enseñanza y, por otro lado, poder generalizar los resultados obtenidos de la muestra al conjunto de la población. A pesar de todo, el motivo que más peso ha alcanzado en la elección de esta metodología ha sido para lograr la máxima objetividad en los resultados y reducir al máximo el sesgo de subjetividad de los investigadores.

Asimismo, se trata de un estudio descriptivo, basado en la observación, análisis de las distintas variables y con carácter sincrónico, estudiando los hechos en un periodo de tiempo en concreto y de corta duración.

Para poder efectuar la investigación, primeramente, se indagó sobre el número de cooperativas de enseñanza en la Ciudad Autónoma de Ceuta. Una vez conocida la cifra de 3 centros cooperativos en total, se procedió a conocer el número de docentes-socios; para ello, se realizaron llamadas telefónicas a los distintos administradores de cada uno de los centros educativos. En total se obtuvo la cifra de 68 docentes-socios en la citada ciudad. En dichas llamadas, a su vez, se explicó a los directivos el objeto de la investigación a desarrollar con el fin de conseguir el consentimiento de cada una de las administraciones para llevar a cabo la recogida de datos, a través de la realización de un cuestionario por cada uno de los cooperativistas.

\subsection{Sujeto de estudio}

La muestra seleccionada para esta investigación comprende la totalidad de docentes-socios cooperativistas $(n=68)$ de la Ciudad Autónoma de Ceuta, de los cuales se obtuvieron datos de 60 de ellos, constituyendo el $88,2 \%$ de la muestra seleccionada.

Los sujetos participantes fueron escogidos por un muestreo dirigido o por conveniencia (Hernández, Fernández y Baptista, 2014) debido a la facilidad para acceder a tales profesionales de la educación. 


\subsection{Instrumento de recogida de datos}

\subsubsection{Elaboración del instrumento}

Para efectuar esta investigación se ha elaborado un cuestionario ad hoc, compuesto por una serie de cuestiones que abarcan todas las variables de la investigación y que permitan dar respuesta a los objetivos planteados (Alaminos y Castejón, 2006). Todos los ítems se presentan planificados y estructurados siguiendo una lógica, con el objetivo de ofrecer unos datos claros y precisos.

Las principales ventajas que presenta este instrumento, siguiendo a autores como Díaz (2001) y Torres, Paz y Salazar (2006), son su reducido coste económico, su fácil aplicación y análisis de los datos, su rapidez en la obtención de información y el escaso esfuerzo que tiene que ejercer el encuestado para ofrecer su opinión.

Particularmente, el cuestionario elaborado para esta investigación está compuesto por 57 preguntas cerradas, con 4 posibles respuestas, que oscilan en un rango de opinión utilizando una escala Likert.

Seguidamente, se procedió a un juicio de expertos para que evaluaran y validaran el cuestionario antes de ponerlo en práctica. Dicho juicio de expertos estuvo compuesto por 3 doctores de la Facultad de Educación, Economía y Tecnología de Ceuta, perteneciente a la Universidad de Granada y, además, por 3 miembros de los equipos directivos de las cooperativas analizadas. La opinión de estos especialistas fue bastante positiva, no teniendo que realizar grandes modificaciones.

El siguiente paso fue comprobar la fiabilidad y la consistencia interna del cuestionario mediante la utilización del estadístico Alfa $(\alpha)$ de Cronbach, obteniendo un resultado de $\alpha=0,8640$, considerado un valor de consistencia alta, ya que dicho valor se encuentra entre el intervalo $0,8<\alpha<1$ (Bisquerra, 2004).

Por último, para hacer aún más sencillo el proceso, al comienzo de cada cuestionario se situaron las instrucciones para cumplimentar correctamente el mismo. En ellas se encuentran el objeto de la investigación, las normas para su realización y los datos para contactar con los investigadores en caso de duda o problema.

\subsubsection{Aplicación del instrumento}

Una vez finalizado el proceso de elaboración del instrumento se efectuó la recogida de datos. Se entregaron los cuestionarios impresos a los directores de las distintas cooperativas de enseñanza, quienes colaboraron plenamente en el proceso de recogida de datos. Se estableció un plazo de 10 días para rellenar los cuestionarios; una vez pasado el plazo, se procedió a la recogida de estos para, posteriormente, realizar el análisis e interpretación de los datos obtenidos.

\subsection{Análisis de los datos}

Para facilitar la tarea de analizar la información que se extraen en bruto de los cuestionarios, existen programas estadísticos específicos que permiten obtener datos de suma importancia para el investigador de cada una de las variables que estructuran el estudio. Para ello se ha utilizado la aplicación Statistical Package for the Social Sciences (SPSS) en su versión 20. Se trata de un software específico de tratamiento estadístico que cuenta con la capacidad para trabajar con grandes bases de datos.

\section{RESULTADOS}

A continuación, se presentan los resultados más significativos y relevantes del estudio para lograr alcanzar los objetivos marcados al comienzo de la investigación.

En la figura 1 se presenta el porcentaje de mujeres y hombres socios de las cooperativas de enseñanza estudiadas. Como se observa, se muestra un amplio y elevado porcentaje de mujeres cooperativistas. Este representa el $75 \%$ del total, frente al escaso $25 \%$ de hombres que desarrollan sus labores docentes y empresariales en las cooperativas de enseñanza de la ciudad. Por lo tanto, se observa dominancia de la mujer sobre el hombre en esta profesión.

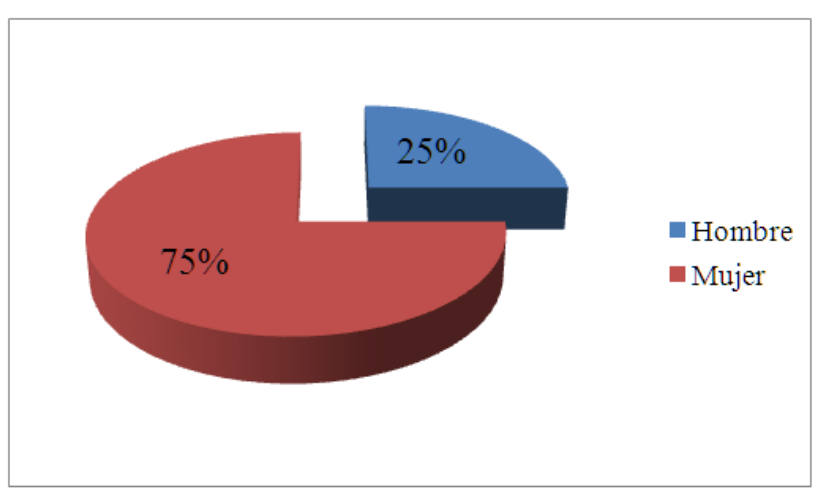

Figura 1. Género de los docentes-socios de las cooperativas de enseñanza.

Con respecto a la figura 2, se representan los diferentes intervalos de edad de los docentes. Como se aprecia, el intervalo que más destacado es el de socios mayores de 50 años, seguido de una ajustada igualdad entre los dos intervalos que le preceden. 


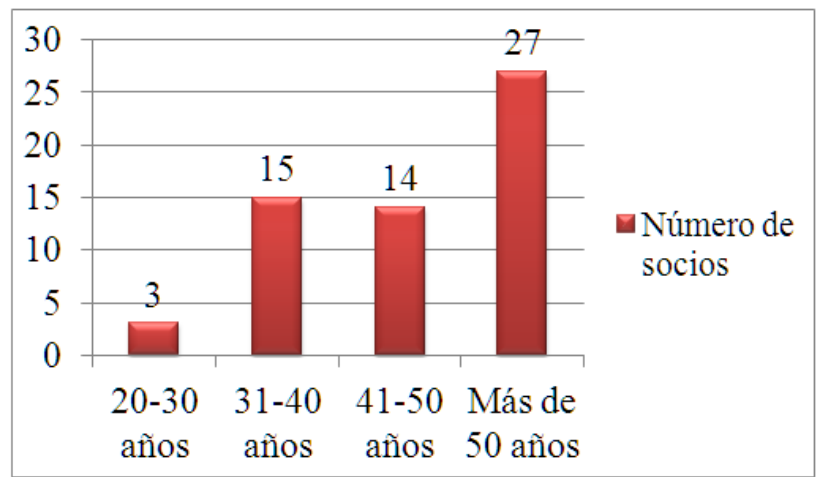

Figura 2. Intervalos de edad de los socios de las cooperativas de enseñanza.

Una amplia mayoría de los docentes-socios (figura 3) que componen estas entidades cooperativas en el ámbito de la educación conocen las características y peculiaridades que caracterizan a este tipo de sociedades, además de su naturaleza y objetivos que persiguen dentro del marco económico.

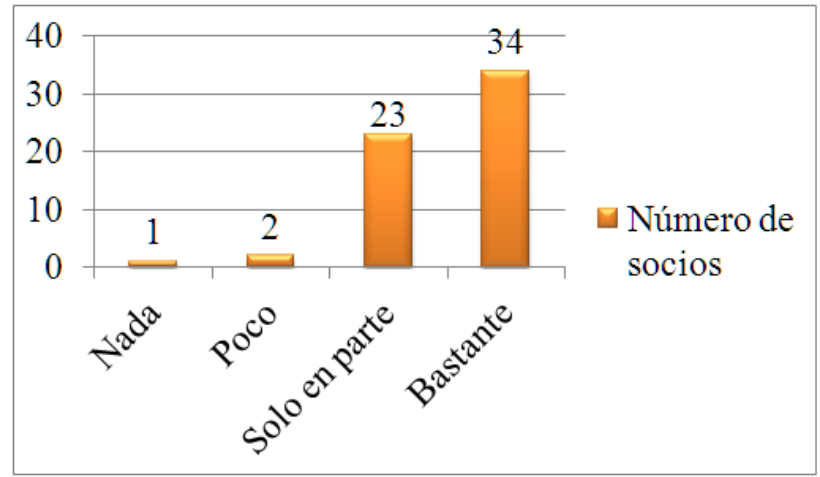

Figura 3. Conocimiento de los docentes-socios sobre las características y peculiaridades de las cooperativas de enseñanza.

En referencia a la figura 4, sobre la consideración y opinión de los socios acerca de la fórmula cooperativa como inversión en un puesto de trabajo docente, la mayoría ( $\mathrm{n}=48$ socios) piensa que resulta bastante positivo realizar un desembolso económico para adquirir una plaza como docente en un centro de enseñanza de índole cooperativa. Seguido de una menor cifra de 8 profesionales que "solo en parte" consideran provechosa tal inversión. Cabe resaltar que solo 2 socios opinan que es "poco" efectiva la operación económica para trabajar como docente en un centro alternativo al sistema público.

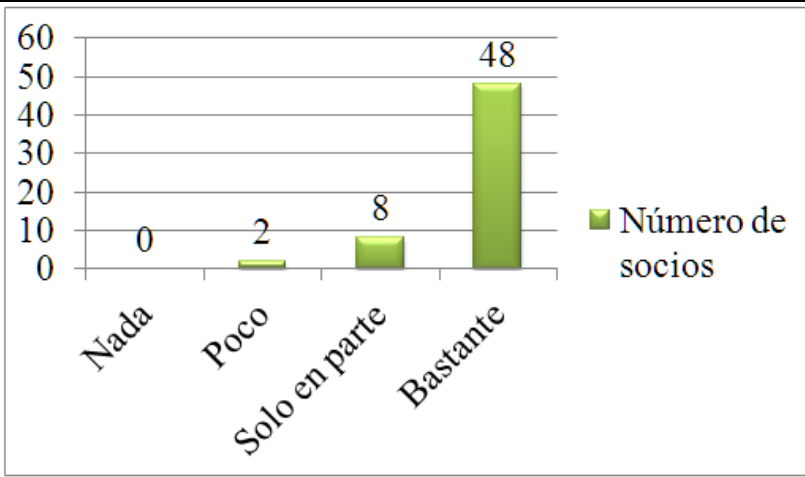

Figura 4. Consideración de los docentes-socios acerca de la fórmula cooperativa como inversión educativa.

En cuanto al conocimiento que disponen los docentes-socios sobre los trámites y procedimientos legales que hay que realizar para la constitución de nuevas cooperativas de enseñanza (figura 5), hay que destacar que más de la mitad de los socios tendrían problemas para iniciar una nueva entidad, ya que apenas tienen conocimientos en esta materia. Solo 9 socios se han manifestado firmes en la idea de que poseen los conocimientos necesarios para emprender el camino hacia la creación de una nueva cooperativa de enseñanza sin tener ningún tipo de problemas a la hora de realizar todas las gestiones que conllevaría tal acción.

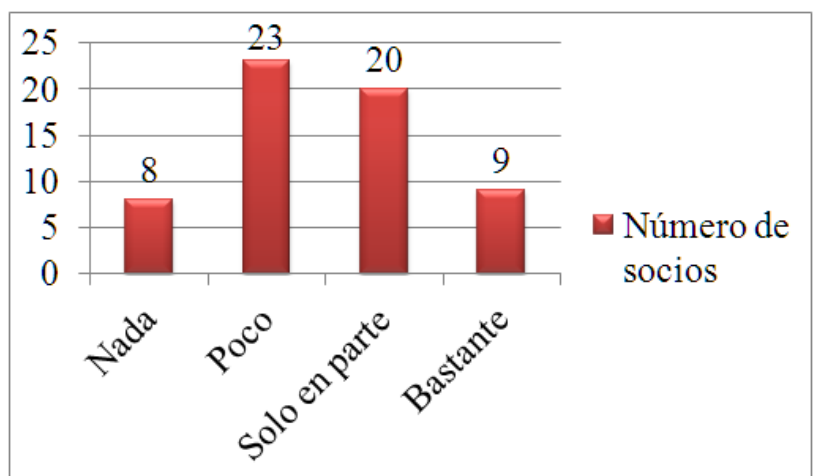

Figura 5. Conocimiento de los socios sobre los trámites y procedimientos legales para crear una nueva cooperativa de enseñanza.

Sobre el fenómeno del liderazgo en las organizaciones sociales (figura 6), la mayoría de los socios han manifestado que conocen el concepto y las características que engloban al término de liderazgo. Solo una escasa cifra de 4 docentes-socios no tiene conocimiento sobre este fenómeno tan relevante en el desarrollo de una entidad. 


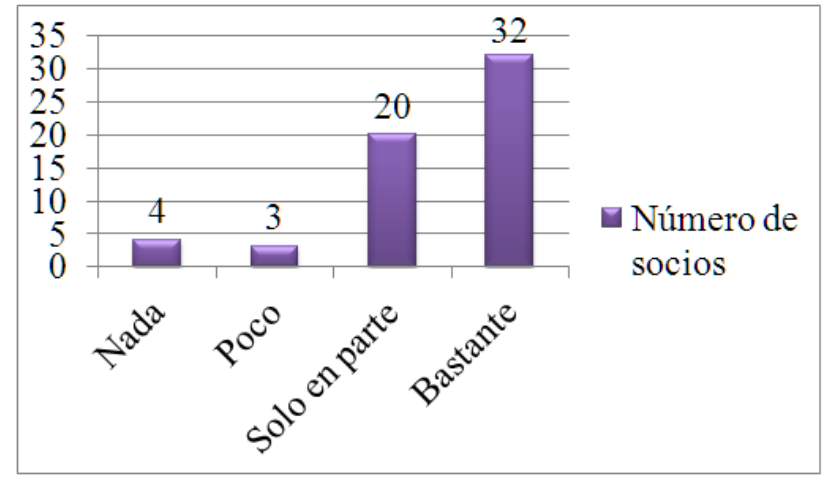

Figura 6. Conocimiento de los socios sobre el concepto de liderazgo.

Además de conocer lo que implica el liderazgo en un grupo de personas, un amplio grupo de socios $(n=53)$ conocen las peculiaridades y habilidades que reúnen las personas para ser auténticos líderes. Apenas 6 socios son los únicos que presentan escasas nociones sobre este concepto (figura 7).

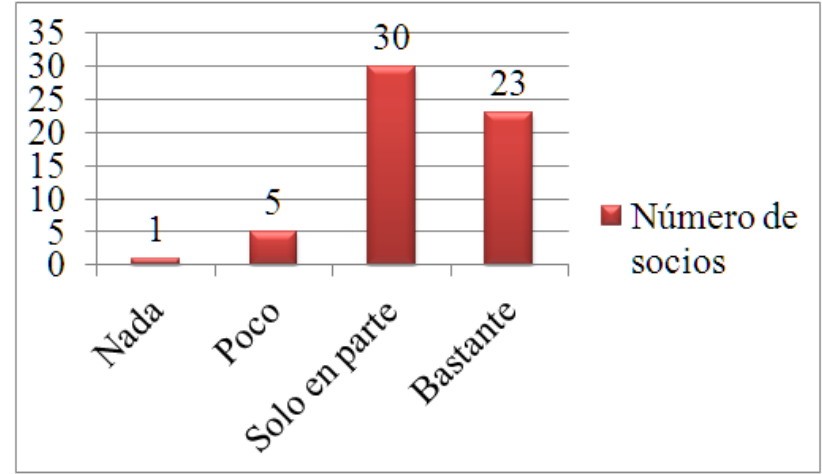

Figura 7. Conocimiento de los docentes-socios sobre las peculiaridades para ser un líder efectivo en la cooperativa.

En la figura 8 se refleja la importancia que otorgan los docentes-socios al fenómeno del liderazgo. Como se ha visto hasta ahora, el liderazgo es considerado por casi todos (a excepción de 7 socios) de suma relevancia en este tipo de empresas basadas en la economía social a nivel educativo.

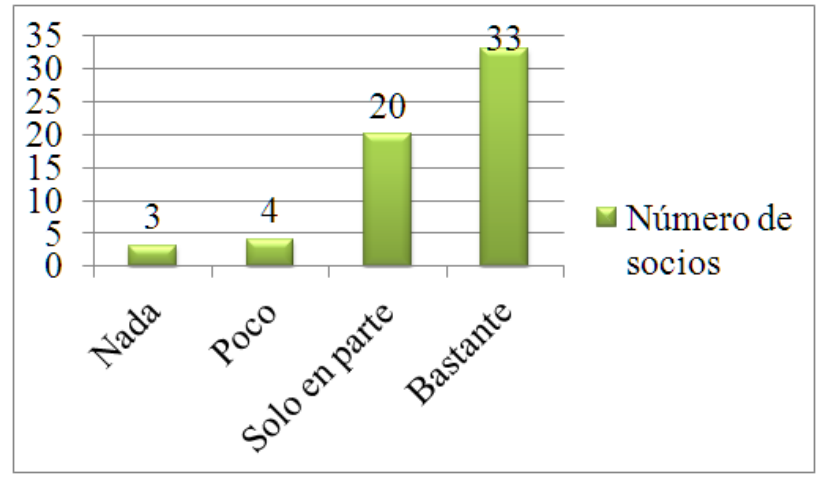

Figura 8. Importancia del liderazgo en las cooperativas de enseñanza.
Para alcanzar la calidad perseguida por toda entidad social resulta primordial establecer no solo perfiles de liderazgo entre los socios que componen el grupo de trabajadores, sino llevar a cabo un sistema de formación continua y permanente durante toda la vida laboral de una persona. La sociedad evoluciona y los profesionales tienen que adaptarse a las nuevas formas metodológicas que condicionan la educación actual. Como se muestra en la figura 9, un alto índice de respuestas $(n=56)$ otorga gran importancia a la formación complementaria a los estudios universitarios.

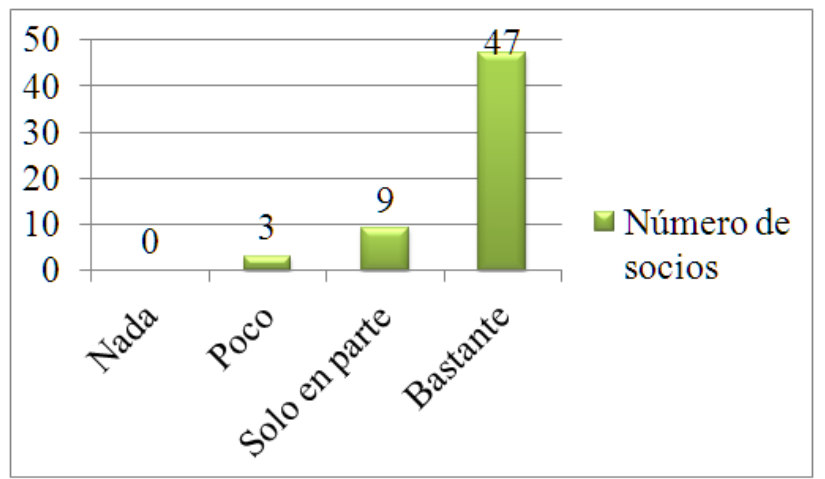

Figura 9. Importancia que otorgan los socios a la formación complementaria.

A pesar de que consideran de suma importancia la formación continua docente, la figura 10 refleja que en materia cooperativa y de ámbito empresarial los socios no poseen demasiado conocimiento, siendo este campo de estudio relevante para la estabilidad, crecimiento y desarrollo como entidad. Solo un pequeño grupo de 6 docentes-socios manifiestan que sí poseen los conocimientos necesarios en esta materia.

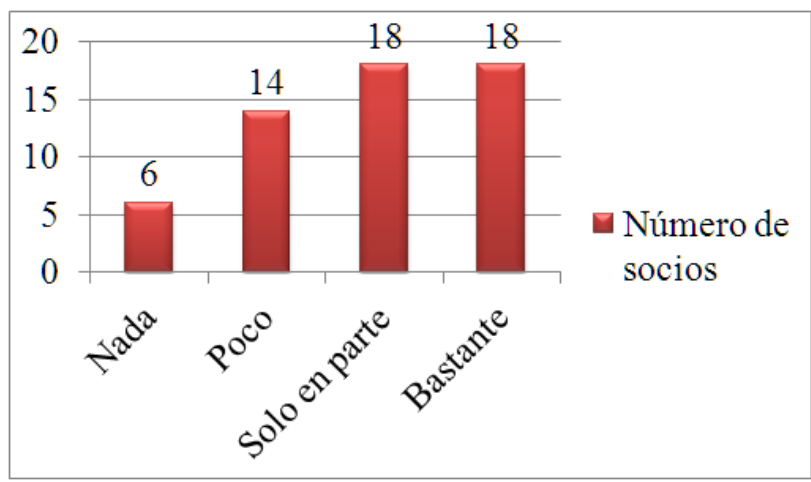

Figura 10. Consideración de la escasez de conocimientos en materia cooperativa y empresarial.

En contraste a la importancia otorgada en la formación permanente que se lleva a cabo para lograr la calidad y la mejora de los servicios educativos y gestión empresarial de estos centros de 
enseñanza, un grupo de 39 docentes-socios realizan entre 1 y 2 cursos de formación al año, siendo apenas 6 socios lo que realizan más de 3 cursos en un periodo lectivo (figura 11).

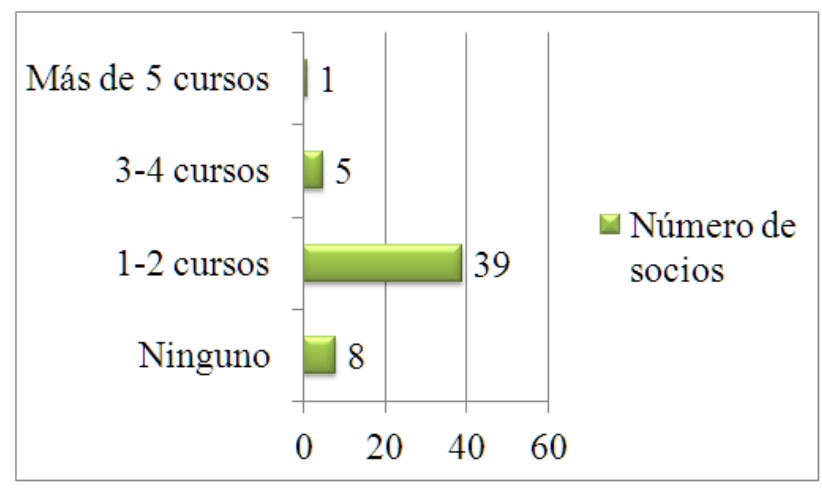

Figura 11. Cursos de formación complementaria realizados por los docentes-socios al año.

\section{DISCUSIÓN}

Los resultados obtenidos revelan que la educación es uno de los sectores de actuación en los que se da el cooperativismo (Eid y Martínez, 2014).

Los profesionales que las integran manifiestan que conocen las peculiaridades y características que engloban a este tipo de entidades sociales, confirmando estudios previos realizados por López (2017).

En cuanto a la consideración de la fórmula cooperativa como inversión en el sector educativo, al igual que Ciruela, Cuadrado y Plaza (2016), Inglada, Sastre y Villarroya (2015) y Sanchís, Campos y Mohedano (2015), se afirma la idea de ser una apuesta de futuro tal acción realizada para desempeñar la labor docente en tiempos de recesión económica y difícil acceso laboral.

En materia de liderazgo, los socios consideran que este fenómeno es un factor fundamental en las organizaciones sociales como afirman Cuevas, Díaz e Hidalgo (2008) y Gutiérrez, Gracia y Quiñonez (2016). Los docentes se muestran conocedores de las características que deben reunir los líderes dentro de una organización para la efectiva dinamización de los distintos agentes que componen las cooperativas de enseñanza como manifiestan López y Fuentes (2018).

Con respecto a la formación continua, los docentes-socios consideran que es un elemento importante para la consecución de los fines marcados, al igual que Bacaicoa y Marín (2002), Gisbert y Lázaro (2015) y González y Barba (2014) obtuvieron en sus estudios; pero la realidad es que los socios de las cooperativas analizadas presentan carencias formativas en el ámbito administrativo y empresarial para dirigir satisfactoriamente este tipo de organizaciones. Por tanto, la calidad que manifiestan autores como Casanova (2016), Cuadrado y Ciruela (2014), Muñoz y Murillo (2010), Prieto (2001) y la estabilidad y futuro como entidad que defienden Melián y Campos (2010) se ve en peligro por la falta de conocimientos $y$ competencias en dicha materia.

\section{CONCLUSIONES}

Tras el estudio realizado se concluye que en este tipo de entidades educativas predomina la existencia de profesionales de la educación de género femenino que deciden emprender en el sector educativo para obtener un puesto de trabajo que le permita desarrollar su vocación docente. Este colectivo presenta una edad avanzada, siendo la mayoría de más de 50 años.

Tales profesionales conocen las características y peculiaridades que reúnen este tipo de sociedades educativas y confían en el cooperativismo como inversión, pero en contraposición a esto, poseen ciertas deficiencias sobre el conocimiento de los trámites y procedimientos legales para crear y gestionar nuevas cooperativas.

En referencia al liderazgo, los docentes-socios presentan nociones sobre tal concepto y conocen las características que tiene que reunir una persona para ser un líder efectivo en la organización. Además de otorgar gran importancia a este fenómeno en este tipo de entidades escolares.

En el plano formativo, consideran relevante los procesos de formación permanente, pero presentan un déficit en el número de cursos realizados al año, puesto que la mayoría no realiza más de 2 cursos, y en el campo de conocimiento de ámbito empresarial.

Con esta investigación se pretende dar a conocer y acercar la realidad que presentan los profesionales que integran las cooperativas de enseñanza en la sociedad actual, reflejando los aspectos más significativos relacionados con el emprendimiento, liderazgo y formación docente.

La principal limitación hallada en este estudio se ha centrado en la cumplimentación de los cuestionarios por parte de los participantes de la muestra.

Como futura línea de investigación se pretende replicar este estudio en la Ciudad Autónoma de Melilla, con la finalidad de establecer una comparación entre los resultados obtenidos en las distintas variables de estudio en ambas ciudades españolas situadas al norte del continente africano. 


\section{REFERENCIAS}

Alaminos, A., y Castejón, J. L. (2006). Elaboración, análisis e interpretación de encuestas, cuestionarios y escalas de opinión. Alicante: Marfil.

Bacaicoa, F., y Marín, M. (2002). La formación permanente del profesorado ¿para qué sirven los cursos de actualización? Revista Fuentes, 3, 32-44.

Bisquerra, R. (2004). Metodología de la investigación educativa. Madrid: La Muralla.

Casal, L., y García, B. (2019). Una experiencia de formación del profesorado para implementar la evaluación entre pares en el campus virtual de la Universidad de Santiago de Compostela. Píxel-Bit. Revista de Medios y Educación, $54,125-144$.

Casanova, M. A. (2016). El diseño curricular como factor de calidad educativa. REICE. Revista Iberoamericana sobre Calidad, Eficacia y Cambio en Educación, 10(4), 7-20.

Ciruela, A. M., Cuadrado, M., y Plaza, J. J. (2016). La economía social como alternativa de autoempleo. El perfil del emprendedor/a en las sociedades laborales andaluzas. REVESCO: Revista de estudios cooperativos, $122,59-85$.

Cuadrado, M., y Ciruela, A. M. (2014). Las sociedades cooperativas y sociedades laborales como motor de desarrollo económico y social: análisis de su impacto socioeconómico en la región de Andalucía. REVESCO: Revista de estudios cooperativos, 115, 57-100.

Cuevas, M., Díaz, F., e Hidalgo, V. (2008). Liderazgo de los directores y calidad de la educación. Un estudio del perfil de los directivos en un contexto pluricultural. Profesorado. Revista de currículum y formación del profesorado, 12(2), 1-19.

Cuñat, R. J. (2015). Las redes como factor clave para la consolidación de nuevas cooperativas de trabajo asociado. REVESCO: Revista de estudios cooperativos, $119,26-52$.

Díaz, V. (2001). Diseño y elaboración de cuestionarios para la investigación comercial. Madrid: ESIC Editorial.

Egido, I. (2010). El acceso a la profesión docente en España en perspectiva europea. Algunas reflexiones orientadas a la mejora de la selección del profesorado. Educación $X X 1,13(2), 47-67$.

Eid, M., y Martínez, F. (2014). El año internacional del cooperativismo. Una aproximación a los desafíos del sector mediante el método Delphi. REVESCO. Revista de Estudios Cooperativos, 116, 103-129.

Ferrera, J. (2013). Educación y liderazgo: una convergencia necesaria. Edetania: estudios y propuestas socioeducativas, 44, 135-150.

Frantz, W., y Fonseca, E. M. (2013). ¿Retorno al movimiento cooperativo? Retos para la economía, la política y la educación. Didácticas Específicas, 9, 5-29.

Fuentes, A. M. (2004). Las cooperativas de enseñanza: (un estudio de las cooperativas de trabajo asociado). Ceuta: Universidad Nacional de Educación a Distancia, Centro Asociado de Ceuta.

Gadea, E., y Atxabal, A. (2015). Las sociedades cooperativas construyen un mundo mejor. REVESCO: Revista de Estudios Cooperativos, 117, 7-11.

Gisbert, M., y Lázaro, J. L. (2015). La formación permanente en competencia digital docente y la mejora de la calidad del centro educativo desde la perspectiva de los docentes: un estudio de caso. Journal of New Approaches in Educational Research, 4(2), 115-122.
González, G., y Barba, J. J. (2014). Formación permanente y desarrollo de la identidad reflexiva del profesorado desde las perspectivas grupal e individual. Profesorado, l(18), 397-412.

Gutiérrez, E., Gracia, J. M., y Quiñonez, J. E. (2016). Liderazgo y gerencia de proyectos educativos y sociales. Dominio de las Ciencias, 2(4), 274-283.

Hernández, R., Fernández, C., y Baptista, M. P. (2014). Metodología de la investigación. Madrid: McGraw Hill.

Inglada, E., Sastre, J. M., y Villarroya, B. (2015). El cooperativismo en la educación. REVESCO: Revista de estudios cooperativos, 118, 122-147.

Ley 27/1999, de 16 de julio, General de Cooperativas. BOE n ${ }^{\circ}$ 170 , de 17/07/1999.

López, J. (2017). El fenómeno del liderazgo en los centros de enseñanza de naturaleza cooperativa. En Alcántara, J.M., Bermúdez, M., Blanco, F.J., y Heredia, J.M. (Eds.), Investigación e innovación en el ámbito universitario. Tendencias ante los retos actuales de la sociedad (pp. 37-46). Madrid: EOS.

López, J., y Fuentes, A. (2018). El liderazgo aplicado a los modelos diferenciados en educación: El caso de las cooperativas de enseñanza. En Gairín, J., y Mercader, C. (Eds.), Liderazgo y gestión del talento en las organizaciones (pp. 169-175). Madrid: Wolters Kluwer.

López, J., Fuentes, A., y Moreno, A. J. (2018). El liderazgo efectivo en los centros concertados de naturaleza cooperativa: Percepciones de sus docentes. Revista actualidades investigativas en educación, 18(3), 1-21.

López, J., Moreno, A. J., y Pozo, S. (2018). Influencia del género y la edad en la formación continua multidisciplinar de los docentes de cooperativas de enseñanza. Revista Innova, 3(8), 42-59.

Maciel, C. (2018). Análisis y valoración de las necesidades de formación de profesores principiantes de Educación Secundaria en Uruguay como base para el diseño de un programa de desarrollo profesional. Educación XX1, 21(1), 63-86.

McMillan, J. H. y Schumacher, S. (2005). Investigación educativa. Madrid: Pearson.

Mehdinezhad, V., y Sardarzahi, Z. (2016). Leadership behaviors and its relation with principals' management experience. Journal of New Approaches in Educational Research (NAER Journal), 5(1), 11-16.

Melián, A., y Campos, V. (2010). Emprendedurismo y economía social como mecanismos de inserción sociolaboral en tiempos de crisis. REVESCO. Revista de estudios cooperativos, 100, 43-67.

Muñoz, M., y Murillo, F. J. (2010). Un balance provisional sobre la calidad en educación: eficacia escolar y mejora de la escuela. REICE. Revista Iberoamericana sobre Calidad, Eficacia y Cambio en Educación, 8(2), 178186.

Prieto, M. (2001). La implantación de la calidad en los centros educativos desde una perspectiva aplicada y reflexiva. Revista Fuentes, 3, 1-2.

Sanchís, J. R., Campos, V., y Mohedano, A. (2015). Factores clave en la creación y desarrollo de cooperativas. Estudio empírico aplicado a la Comunidad Valenciana. REVESCO: Revista de estudios cooperativos, 119, 183-207.

Sans, A., Guàrdia, J., y Triadó, X. M. (2016). El liderazgo educativo en Europa: Una aproximación transcultural. Revista de Educación, 371, 83-106.

Torres, M., Paz, K., y Salazar, F. G. (2006). Métodos de recolección de datos para una investigación. Rev. Electrónica Ingeniería Boletín, 3, 12-20. 
Las cooperativas de enseñanza: un emprendimiento basado en el liderazgo y formación continua docente

Trujillo, J.M. (2015). Las TIC en los centros educativos. EDMETIC, 4(1), 3-8. 